\title{
Intestinal type villous adenoma of the renal pelvis
}

\author{
Jill Hudson, MD; ${ }^{*}$ Thomas Arnason, MD; ${ }^{\dagger}$ Jennifer L.O. Merrimen, MD; ${ }^{\dagger}$ Joseph Lawen, MD, FRCSC ${ }^{*}$
}

*Department of Urology, Queen Elizabeth II Health Sciences Centre, Halifax, NS; †Division of Anatomical Pathology, Department of Laboratory Medicine, Queen Elizabeth II Health Sciences Centre, Halifax, NS

Cite as: Can Urol Assoc J 2013;7:E138-E142. http://dx.doi.org/10.5489/cuaj.257

\section{Abstract}

Intestinal type villous adenomas are uncommon in the genitourinary tract. Most reported cases have been located in the urinary bladder or urachus. Villous adenoma arising in the renal pelvis or ureter is very rare. We present a case of an 81 -year-old female who presented with difficulty voiding and mucosuria. A computed tomography scan identified right-sided hydronephrosis, renal parenchymal atrophy, nonobstructing calculi and a lower pole renal mass. She underwent open right nephrectomy. Histopathologic examination of the kidney revealed an intestinal type villous adenoma of the renal pelvis with high-grade dysplasia and focal areas suspicious for invasive adenocarcinoma. We review the four previously reported cases of intestinal type villous adenoma in the renal pelvis and discuss diagnosis and management of this unusual neoplasm.

\section{Introduction}

Intestinal type villous adenomas are rarely found within the lower urinary tract and even less commonly within the upper urinary tract. We present a case of intestinal type villous adenoma of the renal pelvis with high-grade dysplasia presenting with mucosuria. We discuss the clinical presentation, pathologic features, management, and follow-up of this entity based on our experience and review previous cases.

\section{Case report}

An 81-year-old female presented with a history of difficulty voiding and incomplete bladder emptying. At times, she was only able to void in the supine position. She reported intermittent mucosuria, but denied frequency or dysuria. She was reported by her primary care physician to have had recurrent urinary tract infections, although no positive urine cultures were available to confirm infection. She had been treated with antibiotics with periodic improvement and rapid recurrences of voiding difficulties. She was reported to have a small urethral caruncle. Her medical history included remote poliomyelitis with a resulting gait abnormality, right eye cataract, hypertension and gastro-esophageal reflux disease. Her surgical history included a total abdominal hysterectomy and bilateral salpingo-oophorectomy for benign disease. There was no history of nephrolithiasis. A colonoscopy for symptoms of diverticulitis, performed 1 year prior to the presentation with urinary symptoms, revealed three hyperplastic polyps with no dysplasia. Physical examination revealed that her abdomen was soft and non-tender. No palpable masses or costovertebral angle tenderness were identified.

A uroflow bladder scan demonstrated a maximum flow rate of $17 \mathrm{~mL} / \mathrm{sec}$ with a postvoid residual volume of $125 \mathrm{~mL}$. An initial flexible cystocopy revealed debris in the bladder, but no mucosal abnormality. She was placed on prophylactic trimethoprim and sulfamethoxazole (TMP-SMX) emigpirically. In follow-up, on TMP-SMX therapy, she had multiple negative urine cultures and a normal cystoscopy. However, the intermittent passage of mucous persisted and worsened. Gynecological evaluation showed no abnormalities. As symptoms of difficult emptying persisted, a third cystoscopy, 18 months later, revealed a mucous plug with a solid core in the bladder. We were unable to aspirate the collection and at a subsequent rigid cystoscopy it had passed spontaneously. After an episode of acute urinary retention, more than 2 years after her initial presentation, repeat cystoscopic exam found vermiform mucous strands. These suggested ureteral casts and an upper tract source for the mucous. Abdominal computed tomography (CT) scan confirmed the upper tract abnormality and showed right-sided hydronephrosis to the 

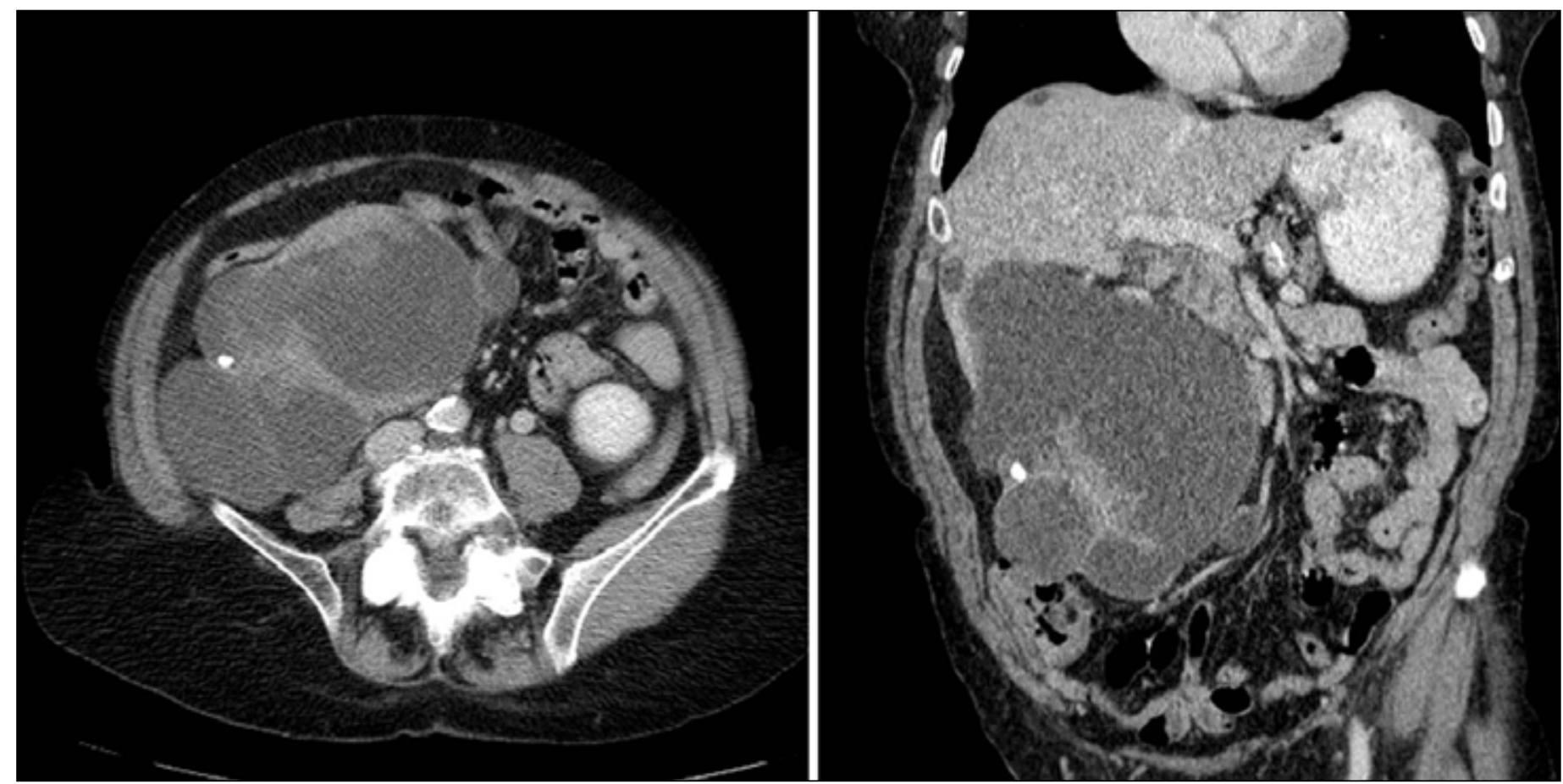

Fig. 1. A computed tomography scan of abdomen and pelvis demonstrating right hydronephrosis and cortical thinning. Calcificiations are visible adjacent to a cystic mass with thickened mural nodules. Axial image on left and coronal image on right.

level of the iliac vessels; atrophic renal parenchyma, two nonobstructing renal calculi and a septated lower pole mass (Fig. 1). She underwent cystoscopy and a right retrograde pyelogram. The ureter was normal to the pelvic brim with good emptying below. We were unable to canulate the collecting system itself above that point. Cause for the obstruction was not evident.

An open right nephrectomy was performed. Spillage of mucous from the renal pelvis into the abdomen occurred due to the large size of the kidney. There was minimal blood loss and copious irrigation was performed. The adrenal gland was left in situ. Follow up abdominal CT scan 6 weeks postoperatively demonstrated no lymphadenopathy or recurrence at the resection site.

The surgical specimen (Fig. 2, part A) weighed $474 \mathrm{~g}$ and measured $20.5 \times 12.0 \times 9.0 \mathrm{~cm}$. The markedly dilated renal pelvis and calices were filled with thick mucin. In the lower pole of the kidney, there was a heterogeneous $8.5-\mathrm{cm}$ mass with papillary areas that was confined within the renal capsule. At the junction of the ureter and the renal pelvis, there was a second obstructing pedunculated $1.0-\mathrm{cm}$ mucinous mass. The renal cortex and medulla in areas uninvolved by the masses were atrophic, measuring 0.1 to $0.2 \mathrm{~cm}$ in thickness.

On light microscopy, the larger mass (Fig. 2, part B) had two components, including mucin pools with fibrosis containing floating segments of low-grade dysplastic intestinal type epithelium and other areas morphologically similar to a colonic villous adenoma. The villi were lined by mucin producing columnar epithelial cells with nuclear crowding, pseudostratification, and hyperchromasia, consistent with intestinal type low grade dysplasia (Fig. 2, part C). In multiple foci (Fig. 2, part D), the villous adenoma displayed more complex architecture with branching and cribriforming, morphologically resembling high grade dysplasia in a colonic adenoma. The neoplasm had an infiltrating pattern and was associated with small mucin pools containing incomplete glands (Fig. 2, part D). Although a desmoplastic response was not identified, we felt that this area was suspicious for a focus of invasive adenocarcinoma. There was no vascular invasion, no infiltration through the renal capsule, and no neoplastic epithelium on the surface of the kidney.

The markedly dilated renal pelvis and calices were predominantly lined by dysplastic intestinal type mucosa. Despite extensive sampling, intestinal metaplasia (without dysplasia) and cystitis glandularis were not identified. The background kidney parenchyma was markedly thinned and scarred with almost complete loss of tubules and glomeruli, in keeping with severe obstructive nephropathy.

Histochemical stains including Periodic acid-Schiff with diastase pretreatment and Alcian Blue confirmed intracytoplasmic and extracellular mucin in the tumour. Immunohistochemical stains applied to sections of the tumour revealed immunopositivity for cytokeratin 7 , cytokeratin 20, and carcinoembryonic antigen (CEA). DNA mismatch repair proteins including MLH1, MSH2, MSH6 and PMS2 were all intact by immunohistochemistry. 

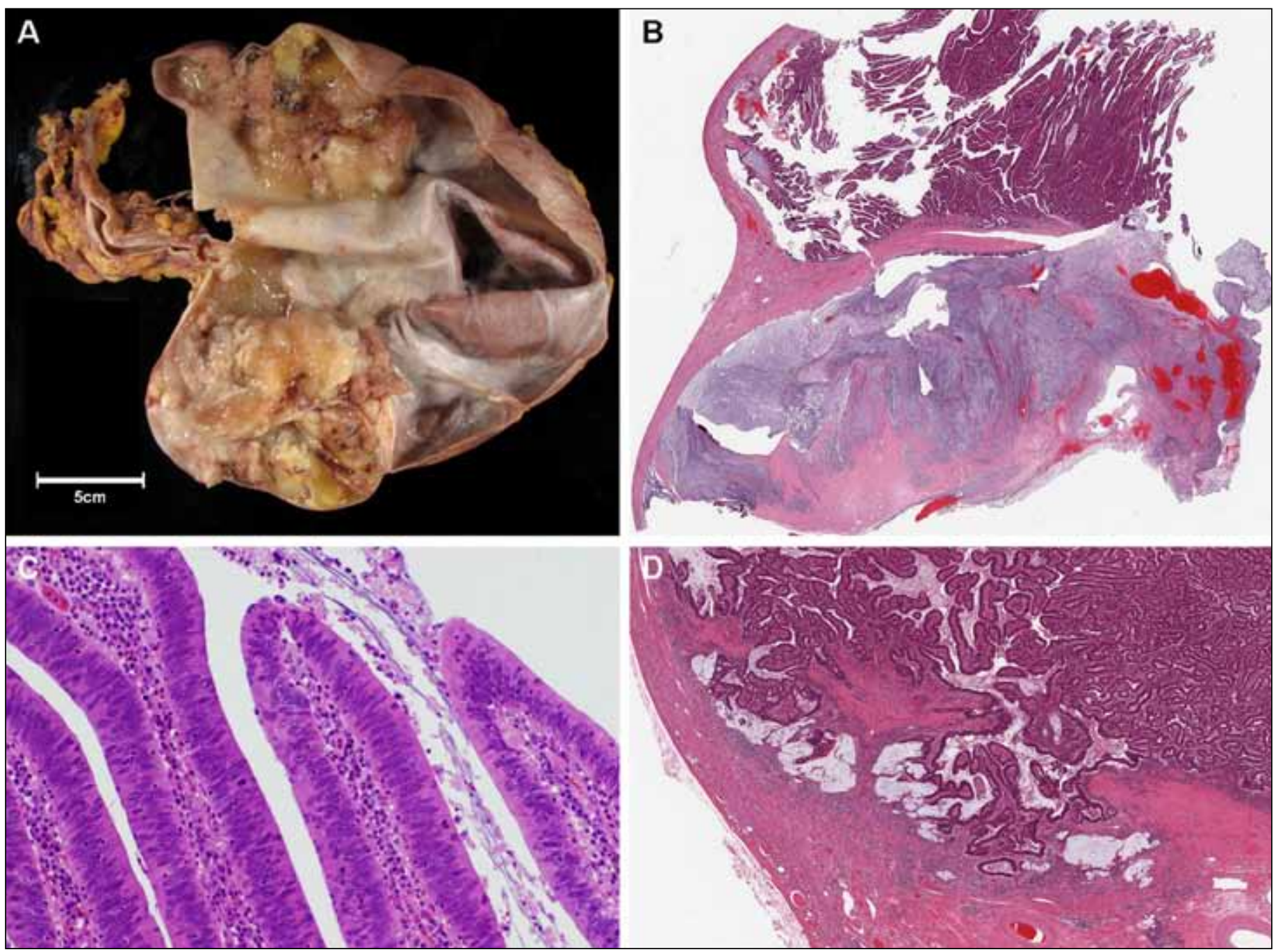

Fig. 2. A: Gross photograph of the markedly dilated kidney showing the mass in the inferior pole; B: Light microscopy (Hematoxylin and eosin [H\&E] original magnification, $\times 5$ ) shows the two components of the mass including villous adenoma (upper) and mucin pooling with fibrosis (lower); C: Villi with dysplastic intestinal type epithelium (H\&E, original magnification, $\times 200$ ); D: Complex architecture with branching and cribriforming, consistent with intestinal high grade dysplasia (right) and infiltrating pattern with mucin pools containing incomplete glands (lower left) (H\&E, original magnification $\times 20)$.

\section{Discussion}

Intestinal type villous adenoma of the genitourinary tract is very uncommon. Most cases arise in the dome, trigone or urachus of the urinary bladder..$^{1-3}$ In the present case, intestinal type villous adenoma arose in the renal pelvis. Only four cases of renal pelvis villous adenoma have been reported in the English-language literature. ${ }^{5-7}$ Bhat and colleagues noted moderate dysplasia without invasion ${ }^{4}$ and Fudge and colleagues demonstrated superficial stromal invasion. ${ }^{5}$ These findings are similar to our own case, which is predominantly a lowgrade villous adenoma, with areas of high-grade dysplasia and microscopic stromal infiltration. The case presented by Fudge and colleagues ${ }^{5}$ and our present case suggest that intestinal type villous adenoma is a precursor to invasive adenocarcinoma in the renal pelvis. A review of adenocarcinomas of the renal pelvis has described villous adenoma like morphology as a common histologic finding in adenocarcinomas of the renal pelvis; this further supports the theory that intestinal type villous adenoma is a precursor to renal pelvis adenocarcinoma. ${ }^{8}$ Consequently, we feel that complete excision of villous adenoma in the renal pelvis by nephrectomy is warranted.

Diagnosis of intestinal-type villous adenoma of the renal pelvis prior to definitive excision is a challenge. Some patients, including the present one, report intermittent mucosuria. Symptoms noted in prior cases of villous adenoma of the renal pelvis include back or flank pain, fever, abdominal discomfort and weight loss. ${ }^{4-6}$ A history of urinary tract infection ${ }^{6}$ and nephrolithiasis ${ }^{4,5}$ have been identified as risk factors. In the more common cases arising in the bladder, patients commonly present with irritative voiding symptoms and hematuria. ${ }^{1}$ 
There are no specific imaging findings on CT scan for villous adenoma of the renal pelvis. In this case and the four previous cases, hydronephrosis and thinning of the renal cortex was identified on $\mathrm{CT}^{4-7}$ In a case of villous adenoma of the ureter, there was obstruction and hydroureternephrosis on both CT and retrograde pyleogram to the L3 level. ${ }^{9} \mathrm{~A}$ mass, which may include cystic changes, is also common. Non-obstructing stones are also identified on CT in three of the five reported cases, including the present one. ${ }^{4,9}$ None of the reports prior to this one identified a cystoscopic finding of vermiform mucous strands within the bladder, which is indicative of an upper tract source, similar to the finding of vermiform blood clots. ${ }^{10}$ While the presence of mucous may suggest intestinal-type villous adenoma, this can also be found in association with pseudomyxoma peritonei, xanthogranulomatous pyelonephritis and local invasion from gastrointestinal malignancy. ${ }^{6,11}$ Therefore, definitive diagnosis relies on light microscopy.

The pathogenesis of villous adenomas in the urinary tract has been postulated to arise from a background of intestinal metaplasia in the urothelium. ${ }^{1,6}$ Intestinal metaplasia of the urinary tract is often associated with chronic inflammation, in particular urinary tract infection, stones, or chemical injury. ${ }^{1,4}$ In the pathogenesis of both colorectal adenocarcinoma and urothelial carcinoma of the upper urinary tract, there has been recent interest in DNA mismatch repair proteins. Patients with the hereditary non-polyposis colon cancer syndrome (HNPCC) or Lynch syndrome are known to have a 14- to 75-fold increased risk of upper urothelial tract carcinomas. ${ }^{12}$ The molecular pathogenesis of these inherited tumours is related to defects in the genes encoding the DNA mismatch repair proteins MLH1, MSH2, MSH6 and PMS2. ${ }^{13}$ Sporadic and inherited defects in the mismatch repair genes are also implicated in about $15 \%$ of colorectal adenocarcinomas. ${ }^{12}$ Given that this villous adenoma has the unusual combination of location in the upper urothelial tract and morphologic similarity to colorectal dysplasia and adenocarcinoma, we hypothesized that a defect in mismatch repair proteins may be present. However, when immunohistochemical markers for mismatch repair proteins were applied to sections the tumour, intact nuclear staining was identified. This finding suggests that the mismatch repair protein and microsatellite instability pathway is not pathogenic in this case. Testing of mismatch repair protein expression has not been previously reported in a villous adenoma of the upper urothelial tract.

Prognosis for intestinal type villous adenomas of the genitourinary tract is not well-studied. While locally recurrent and metastatic adenocarcinomas have not been observed in association with villous adenoma of the renal pelvis, this type of aggressive behaviour has been observed in the bladder. In their series of 23 patients with bladder villous adenomas, Cheng and colleagues found that $33 \%$ had coexistent invasive adenocarcinoma. ${ }^{1}$ In this series, one patient with coexistent invasive carcinoma (treated with radiation) developed a lung metastasis and another patient had multiple local recurrences. However, none of the patients with isolated villous adenoma had a recurrence or metastatic adenocarcinoma over a mean follow-up of 9.9 years. In another series of 18 patients with villous adenoma of the bladder, $33 \%$ of patients had an associated adenocarcinoma. ${ }^{3}$ Two of these patients with associated invasive adenocarcinoma went on to have distant metastases (to lung and small bowel) despite radical surgery. ${ }^{3}$

Given its rarity, there are no guidelines for follow-up or adjuvant treatment of intestinal-type villous adenoma in the renal pelvis following resection. Since the surgical resection margins were negative and there was no evidence of distant metastasis, we felt that there was no role for further intervention in the present case. For urothelial carcinoma of the renal pelvis after a complete resection, the typical follow-up is controversial. However, given its morphology, this tumour may have biologic behaviour which more closely resembles colon adenocarcinoma. In that disease, after a complete resection, the recommended follow-up is CEA every 3 to 6 months for 3 years, with chest/abdomen/ pelvis CT annually for 3 years and colonoscopy yearly. ${ }^{14}$ In colonic adenocarcinoma, CEA is a useful serum tumour marker to identify recurrence. Given that this tumour, like many other villous adenomas and associated adenocarcinomas reported in the lower urinary tract, is positive for CEA by immunohistochemical staining of formalin fixed tissue, there may be a role for serum CEA as a tumour marker. However, immunohistochemistry on tumour tissue is not necessarily predictive of serum CEA levels. Unfortunately, the serum CEA level was not measured prior to surgical resection of the tumour in this case. Serum CEA levels have not been reported in other series and reports of tumours of this type, but would be of interest to investigate in future cases. For this patient, we have arranged CT scans of the chest, abdomen and pelvis in 6 months in addition to the CT scan already performed.

\section{Conclusion}

Intestinal type villous adenoma of the renal pelvis is challenging to diagnose prior to surgical resection but symptoms such as mucosuria, dysuria, flank and abdominal pain, as well as a history of recurrent urinary tract infections and urolithiasis, may be present. On CT scan, features, such as hydronephrosis, hydroureter and renal cortical thinning, are often present. Vermifom mucous strands on cystoscopy and obstruction during retrograde pyelogram may be identified. The finding of invasive adenocarcinoma in association with previous cases of intestinal type villous adenoma suggests that this neoplasm is a precursor to malignancy and 
Hudson et al.

definitive surgical resection with nephrectomy is warranted. Although experience is limited with these neoplasms, the prognosis appears to be good following resection of an isolated villous adenoma, particularly if the experience from the lower urinary tract can be applied to upper urinary tract villous adenomas. The long-term follow-up strategy, including the role (if any) for imaging and tumour markers, such as CEA, remains to be defined.

\section{References}

1. Cheng L, Montironi R, Bostwick D. Villous Adenoma of the Urinary Tract: A Report of 23 Cases, Including 8 With Coexistant Adenocarcinoma. Amer J Surg Pathol 1999;23:764-31. http://dx.doi. org/10.1097/00000478-199907000-00003

2. Mazzucchelli R, Scarpelli M, Montironi R. Mucinous adenocarcinoma with superficial stromal invasion and villous adenoma of urachal remnants: a case report. J Clin Path 2003;56:465-7. http://dx.doi. org/10.1136/icp.56.6.465

3. Seibel J, Prasad S, Weiss R, et al. Villous adenoma of the urinary tract: a lesion frequently associated with malignancy. Hum Pathol 2002;33:236-41. http://dx.doi.org/10.1053/hupa.2002.31293

4. Bhat $S$, Chandran V. Villous adenoma of the renal pelvis and ureter. Indian J Urol 2010;26:598-9. http://dx.doi.org/10.4103/0970-1591.74478
5. Fudge KG, Glicklich D, Golestaneh L, et al. Villous Adenoma in the Native Kidney of a Renal Transplant Recipient. Transplantation 2006;81:638. http://dx.doi.org/10.1097/01.tp.0000188617.65532.1d

6. Park S, Meng MV, Greenberg MS, et al. Muconephrosis. Urology 2002;60:344. http://dx.doi. org/10.1016/S0090-4295(02)01707-7

7. Tamboli P, Ro JY. Villous adenoma of urinary tract: a common tumour in an uncommon location. Adv Anal Pathol 2000;7:79-84. http://dx.doi.org/10.1097/00125480-200007020-00002

8. Spires SE, Banks ER, Cibull ML, et al. Adenocarinoma of renal pelvis. Arch Pathol Lab Med 1993:117:115660.

9. Shih CM, Wu SC, Lee CC, et al. Villous Adenoma of the Ureter with Manifestation of Mucus Hydronephrosis. J Chin Med Assoc 2007;70:33-5. http://dx.doi.org/10.1016/S1726-4901 (09)70298-9

10. Gerber GS, Brendler CB. Evaluation of the Urologic Patient: History, Physical Examination, and Urinalysis. Campbell-Walsh Urology. Ed Wein AJ, Kavoussi LR, Novick AC, Partin AW, Peters CA. 201 1; ch.3.

11. Vinaya BS, Gayathri P, Amonkar JR, et al. Mucinous adenocarcinoma of the renal pelvis with pseudomyxoma peritonei. Indian J Pathol Microbiol 2008;51:536-7. http://dx.doi.org/10.4103/03774929.43753

12. Ericson $K$, Nilbert $M$, Bladström $A$, et al. Familial risk of tumors associated with hereditary non-polyposis colorectal cancer: a Swedish population-based study. Scand I Gastroenterol 2004;39:1259-65.

13. Amira N, Mourah S, Rozet F, et al. Non-invasive molecular detection of bladder cancer recurrence. Int J Cancer 2002;101:293-7.

14. NCCN. Clinical Practice Guidelines in Oncology: Colon Cancer. 2011:3.

Correspondence: Dr. Joseph Lawen, Department of Urology, Queen Elizabeth II Health Sciences Centre, Suite 620, 5991 Spring Garden Rd, Halifax, NS B3H 1Y6; joseph.lawen@dal.co 